\title{
Autophagy-induced RelB/p52 activation mediates tumour-associated macrophage repolarisation and suppression of hepatocellular carcinoma by natural compound baicalin
}

\author{
H-Y Tan ${ }^{1,5}$, N Wang ${ }^{1,5}$, K Man $^{2}$, S-W Tsao ${ }^{3}$ C-M Che ${ }^{4}$ and Y Feng ${ }^{\star, 1}$
}

The plasticity of tumour-associated macrophages (TAMs) has implicated an influential role in hepatocellular carcinoma (HCC). Repolarisation of TAM towards M1 phenotype characterises an immune-competent microenvironment that favours tumour regression. To investigate the role and mechanism of TAM repolarisation in suppression of HCC by a natural compound baicalin, Orthotopic HCC implantation model was used to investigate the effect of baicalin on HCC; liposome-clodronate was introduced to suppress macrophage populations in mice; bone marrow-derived monocytes (BMDMs) were induced to unpolarised, M1-like, M2like macrophages and TAM using different conditioned medium. We observed that oral administration of baicalin $(50 \mathrm{mg} / \mathrm{kg})$ completely blocked orthotopic growth of implanted HCC. Suppression of HCC by baicalin was diminished when mice macrophage was removed by clodronate treatment. Baicalin induced repolarisation of TAM to M1-like phenotype without specific toxicity to either phenotype of macrophages. Baicalin initiated TAM reprogramming to M1-like macrophage, and promoted pro-inflammatory cytokines production. Co-culturing of HCC cells with baicalin-treated TAMs resulted in reduced proliferation and motility in HCC. Baicalin had minimal effect on derivation of macrophage polarisation factors by HCC cells, while directly induced repolarisation of TAM and M2-like macrophage. This effect was associated with elevated autophagy, and transcriptional activation of RelB/p52 pathway. Suppression of autophagy or RelB abolished skewing of baicalin-treated TAM. Autophagic degradation of TRAF2 in baicalin-treated TAM might be responsible for RelB/p52 activation. Our findings unveil the essential role of TAM repolarisation in suppressive effect of baicalin on HCC, which requires autophagy-associated activation of RelB/p52.

Cell Death and Disease (2015) 6, e1942; doi:10.1038/cddis.2015.271; published online 22 October 2015

Liver cancer is the second most fatal cancer worldwide, with mortality rate of over $84 \%$ within 5 years. ${ }^{1}$ Of 782000 cases reported worldwide in 2012, China alone has accounted for more than $50 \%$ of the total incidences. Hepatocellular carcinoma (HCC) is the major occurring liver cancers, which represents $70-85 \%$ of cases among all primary liver cancers. ${ }^{2}$ Our recent study postulated that poor prognosis of $\mathrm{HCC}$ is associated with high level of tumour-associated macrophages (M2) markers and M2 macrophages further enhanced HCC invasiveness. $^{3}$ Injection of M2 macrophages could significantly promote tumour growth in orthotopic HCC-implanted mice, which further demonstrated the pro-tumoral role of M2 macrophage in HCC. Reprogramming of TAM away from M2-like, towards M1-like phenotype in tumour microenvironment by FDA-approved anti-HCC drug sorafenib suppressed hepatoma cell proliferation. ${ }^{4}$ These studies suggested the possibility of targeting TAMs as a potential immunemodulating strategy in HCC.
Recent studies increased attention on the plasticity of macrophage in disease progression and control. Under pathological and pharmacological conditions, macrophages acquire distinct phenotypic characteristics through different activation mechanisms. The classical activated macrophage (M1-like), exhibits pro-inflammatory properties by expressing and secreting pro-inflammatory molecules including TNF-a, IL6, IL12, IL1, Type I IFN $\gamma$, CXCL1-3, CXCL-5 and CXCL8-10; while macrophage could be alternatively activated to M2-like phenotype, which expresses anti-inflammatory factors such as IL $10 .^{5} \mathrm{M} 1$-like macrophage, which strongly expresses CD86, iNOS and MHC-II, is distinguishable in phenotype from M2-like macrophage that has a higher level of CD206, CD163, YM1 and Fizz1. ${ }^{6}$ Tumour-associated macrophages (TAMs) are a population of macrophages in tumour microenvironment, which functions in promoting cancer cell proliferation and invasion, regulating tumour neovascularization as well as cytotoxic T-cell function. ${ }^{7,8}$ TAMs are predominantly M2 phenotype and exhibits immunosuppressive function. M2

\footnotetext{
${ }^{1}$ School of Chinese Medicine, The University of Hong Kong, Hong Kong, China; ${ }^{2}$ Department of Surgery, The University of Hong Kong, Hong Kong, China; ${ }^{3}$ Department of Anatomy, The University of Hong Kong, Hong Kong, China and ${ }^{4}$ State Key Laboratory of Synthetic Chemistry, Chemical Biology Center and Department of Chemistry, The University of Hong Kong, Hong Kong, China

*Corresponding author: Y Feng. School of Chinese Medicine, The University of Hong Kong, 10 Sassoon Road, Pokfulam, Hong Kong, China. Tel: +852 25890482; Fax: +852 28725476; E-mail: yfeng@hku.hk

${ }^{5}$ These authors contributed equally to this work.

Abbreviations: TAM, tumour-associated macrophages; HCC, hepatocellular carcinoma; BMDM, bone marrow-derived monocytes; MR, mannose receptor; iNOS, inducible nitric oxide synthase; TNF- $\alpha$, tumour necrosis factor- $\alpha$; IL12, interleukin-12; IL10, interleukin-10; TGF- $\beta$, tumour growth factor- $\beta$; TSN, tumour supernatant; LC3, microtubule-associated protein 1 A/1B-light chain 3; Atg5, autophagy protein 5; TRAF, TNF receptor-associated factor; NF- $\kappa$ B, nuclear factor kappa-B Received 24.5.15; revised 06.8.15; accepted 24.8.15; Edited by GM Fimia
} 
macrophages tend to secrete pro-tumoral cytokines that support tumour growth and suppress T-cell proliferation. Depending on the tumour environment, TAMs phenotype would be reprogrammed to $\mathrm{M} 1$-like that are characterised for its antigen-presenting property and pro-inflammatory function, favours tumour regression. ${ }^{9,10}$

In particular, the role of nuclear factor-kappa B (NF- $k \mathrm{~B})$ in TAMs is enormously studied in recent years. NF- $\kappa \mathrm{B}$ transcription factor is composed of five protein members including p100/p52, RelB, p105/p50, RelA and c-Rel. ${ }^{11}$ Formation and regulation of transcription activities of NF-KB dimers could be achieved by both canonical and non-canonical pathways. Although NF- $\kappa$ B activation is generally considered to trigger pro-inflammation, it is still not conclusive whether it is necessarily in regulating the polarisation of TAMs. In fact, the role of NF-KB in TAMs skewing seems to be controversial across different studies. It was shown that NF- $k$ B activity was suppressed in TAMs co-cultured with ovarian cancer stem cells, which is responsible for the M2 macrophages skewing; ${ }^{12}$ while in ATP-binding cassette transporter G1 (ABCG1)knockout TAMs, NF- $k$ B activity was increased and TAMs exhibited M1 phenotypes and direct toxicity to tumor cells. ${ }^{13}$ Devaraj and colleagues showed that C-reactive protein could repolarise $M 2$ macrophages to $M 1$ phenotype, but it fails to prime those M2 macrophages with inactive NF- $\kappa B^{14}$ Combination treatment of TGF- $\beta$ inhibitor with TLR7 ligand on TAMs leads to M1 reprogramming and associated with obvious nuclear translocation of NF-kB; ${ }^{15}$ TLR9 ligand combined with $\mathrm{IL}-10$ receptor antibody and CCL16 may activate NF- $\mathrm{BB}$ and redirect TAMs towards $\mathrm{M} 1$ phenotype. ${ }^{16}$ Treatments were also designed in targeting on NF- $\kappa$ B in TAMs. A small molecule azithromycin inhibits TLR4-related NF- $k B$ activity, which leads to reduction of $\mathrm{M} 1$ and increase of $\mathrm{M} 2$ cytokines; ${ }^{17}$ TAMs challenged with zoledronic acid achieved M1 phenotypes with increase of NF- $k$ B-inducible M1 cytokines expression; ${ }^{18}$ miRNAs-containing exosomes derived from Epigallocatechin gallate-treated tumour cells could be absorbed by TAMs, and led to elicited NF- $\kappa \mathrm{B}$-associated M1 skewing. ${ }^{19}$ But the role of NF- $\kappa \mathrm{B}$ in TAM polarisation is far more complicated and not just depends on the expression level of NF- $\kappa$ B components. It was found that p50-knockout TAMs or TAMs with RNA interference against p50 normally produce M1 cytokines, and reduced expression of p50 may switch M2-like macrophages to M1 phenotype. ${ }^{20,21}$ However, overexpression of p50 was also suggested to defect NF- $k B$ pathway and led to M2 phenotype in cancer. ${ }^{22,23}$ It was also observed that activation of $\mathrm{IKK} \beta / \mathrm{NF}-\kappa \mathrm{B}$ is required for maintaining $M 2$ phenotype. ${ }^{24}$ Prostate cancer cell-derived CCN3 induced NF- $k B$ activation in macrophages and skewed TAMs to M2 phenotypes; ${ }^{25}$ an in vivo NF- $\kappa$ B decoy may cause TAMs polarisation to M2 phenotype. ${ }^{26}$ All the above studies revealed the controversy of $\mathrm{NF}-\kappa \mathrm{B}$ activation in TAMs polarisation and it is somehow considered to be highly dependent on different tumour types and experimental approaches. ${ }^{11}$ Chan et al. ${ }^{27}$ even demonstrated that NF- $\kappa \mathrm{B}$ activity is responsible for both M1- and M2-cytokines expression in human cytomegalovirus-infected macrophages. The nature of stimulus and physiological content may determine the role of NF- $\kappa \mathrm{B}$ in TAMs polarisation. ${ }^{23}$
Baicalin is a natural flavonoid present in several medicinal plants including Scutellaria baicalensis Georgi. Few studies have revealed the anti-tumour action of baicalin with in vitro cellular HCC models. ${ }^{28,29}$ It was also reported that baicalin may be an immunomodulatory agent by affecting Th17 cells ${ }^{30}$ and dendritic cells. ${ }^{31}$ It was highlighted that immune response may be boosted up by baicalin treatment via increased expressions of IFN $\gamma$ and IL12, which are the essential factors for activating all types of acquired immune response. ${ }^{32}$ This prompts us to take an insight into the action and mechanism of baicalin for potential cancer therapy. We investigated the impact of baicalin on repolarisation of TAM and its role in mediating $\mathrm{HCC}$ inhibition, as well as elaborated the mechanism underlying baicalin-induced autophagy in regulating TAM repolarisation.

\section{Results}

Inhibition of orthotopic HCC growth by baicalin requires presence of macrophages. Few previous studies reported that baicalin may exhibit anti-tumour effect on HCC cells in vitro, ${ }^{33,34}$ but investigation on the action of baicalin in vivo and its underlying mechanism remains scanty. To systematically evaluate the anti-tumour action of baicalin on $\mathrm{HCC}$, we introduced an orthotopic HCC-implanted murine model by embedding small cubes of subcutaneously grown tumour onto the right lobe of mice liver, which has been reported in our previous studies, ${ }^{33-36}$ followed by oral administration of baicalin $(50 \mathrm{mg} / \mathrm{kg})$ every alternate day after 1 week of transplantation. Body weight of mice was monitored once per week and there was no weight loss among the groups after 5 weeks of intervention (Supplementary Figure S1). Five-week treatment of baicalin could completely inhibit orthotopic HCC growth in mice, while relapse of tumour was observed in mice with macrophage removal (Figure 1b). The tumour size in macrophage-depleted mice with baicalin was not as large as that in macrophage-presenting mice without baicalin treatment; and significant relapse of tumour in macrophage-depleted mice in comparison to macrophagepresenting mice treated with baicalin revealed that macrophage is involved in mediating the anti-tumour effect of baicalin (Figure 1c). Immunohistochemistry staining with F4/80 antibody showed successful removal of macrophage by liposomal clodronate treatment, and baicalin did not significantly increase the residence of macrophage in liver. All these indicated that the role of macrophage in mediating anti-tumour effect of baicalin is independent to the increase of macrophages infiltration (Figure 1d). This was further evidenced by the observation that there was no significant increase in inflammatory monocyte in bone marrow or circulating system of baicalin-treated mice (Figures 1e and f). Our results reveal that macrophage has a positive role in regulating the anti-tumour effect of baicalin in HCC.

Baicalin skews macrophages away from M2-like, towards M1-like phenotype. A plenty of studies have revealed that carcinogenesis involves a process of macrophage infiltration into tumour microenvironment followed by polarisation of macrophage by tumour-favouring factors. ${ }^{37}$ Infiltrated macrophages are programmed to anti-inflammatory 


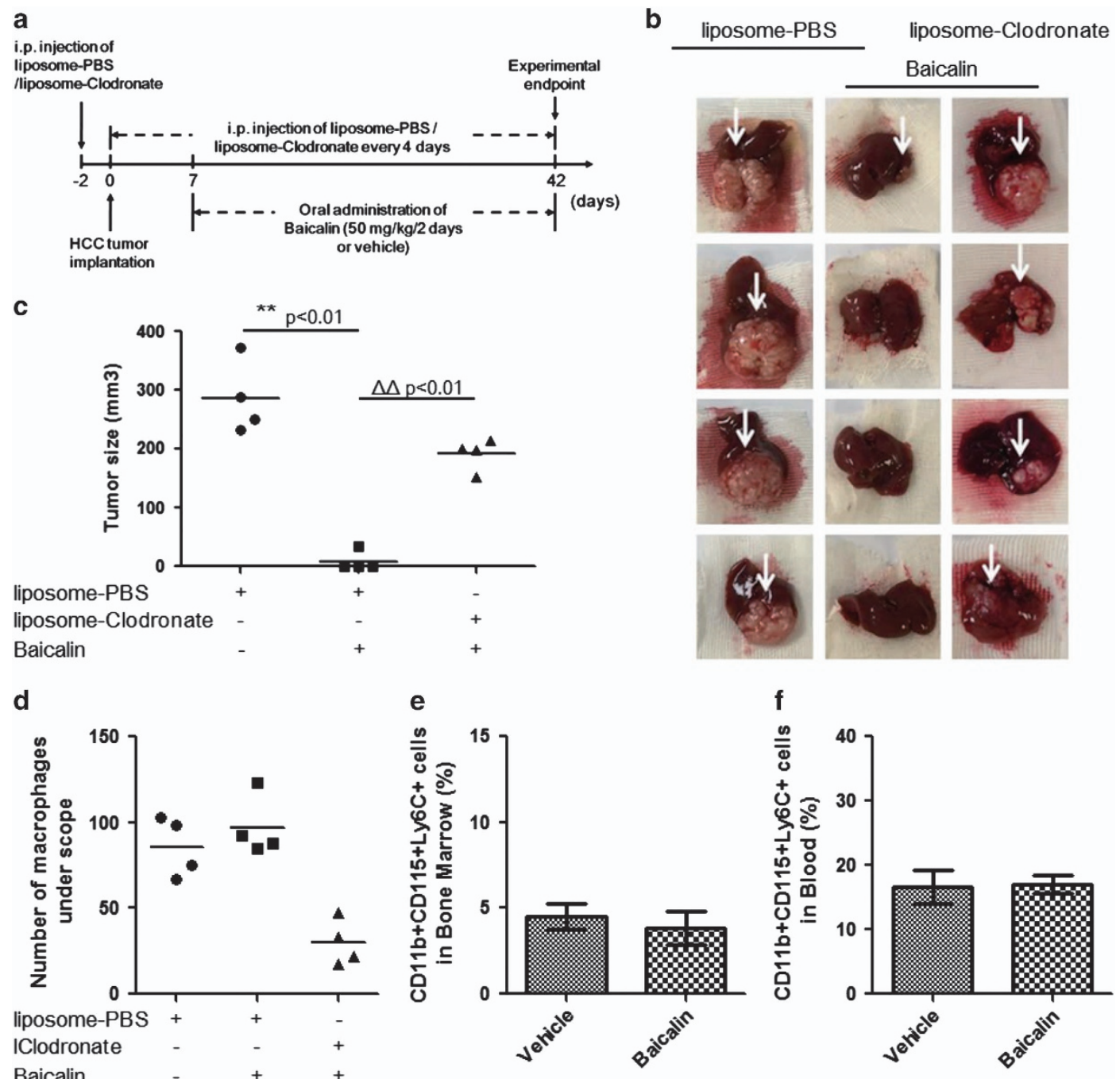

Figure 1 Inhibition of orthotopic HCC growth by baicalin requires presence of macrophages. (a) Schematic presentation of experimental design. (b) Removal of macrophage from mice completely blocked the anti-tumour effect of baicalin. Orthotopic HCC tumour growth in mice $(n=4)$ with baicalin treatment was not obvious, whereas removal of macrophage by intraperitoneally injecting clodronate liposome resulted in relapse of hepatic tumour. (c) The size calculation of hepatic tumour. ${ }^{* \star} P<0.01$, compared with liposome control group without baicalin treatment; ${ }^{\Delta \Delta} P<0.01$, compared with liposome control group with baicalin treatment. (d) The amount of intrahepatic tumour was slightly increased upon baicalin treatment without significance, while presence of clodronate liposome remarkably reduced hepatic macrophage. (e) Baicalin treatment did not potently induce production of inflammatory monocytes in bone marrow. (f) Baicalin treatment did not elevate inflammatory monocytes populations in circulating system

M2-like phenotype, which protects tumour cells from immune surveillance. ${ }^{38}$ M1-like macrophages produce pro-inflammatory cytokines (TNF- $a$, IL12, iNOS, etc), which are against tumour cell growth, whereas M2-like phenotype secrets a series of tumour-favouring factors (IL10, TGF- $\beta$, Arginase1, etc) that promote tumour expansion. ${ }^{39}$ To examine if baicalin could re-programme intrahepatic macrophages towards M1-like phenotype, we measured the presence of $\mathrm{M} 1 / \mathrm{M} 2$-like macrophages in the liver of baicalin-treated mice. Baicalin treatment increased the $\mathrm{iNOS}^{+}$ M1-like macrophages population while significant reduction of $\mathrm{MR}^{+} \mathrm{M} 2$-like population in liver tissue of mice (Figure 2a). We also found that baicalin exhibited no specific cytotoxicity to M1/M2-like macrophages (Figure 2a), which indicated that the effect of baicalin on intrahepatic macrophages phenotype differentiation is independent to cell viability. To further classify the phenotype of baicalin-treated macrophages, we introduced two cell surface markers to distinguish M1-like phenotype from M2. M1-like macrophage possessed relatively higher expression of CD86 while M2-like macrophage highly expressed CD206 (Figure 2c). It was shown that after 7 days of differentiation with HCC tumour supernatant (TSN), the TSN-derived TAMs exhibited high expression of CD206 with relatively low CD86; indicating TAMs are prone to M2-like phenotype (Figure 2d). The 48-h treatment of baicalin re-skewed the TAMs away from M2-like, towards M1-like phenotype dose-dependently (Figure 2d). Consistent with the phenotypic analysis, we observed upregulation of the pro-inflammatory genes, TNF- $a$ and IL12, while downregulation of IL10 and Arginase 1, the anti-inflammatory genes in baicalin-treated $\mathrm{F} 4 / 80^{+}$TAMs (Figure 2e). Baicalin-treated macrophage cells also secreted higher level of proinflammatory cytokines (IL6 and TNF-a) while lower level of IL10 (Figure 2f), which revealed TAM reprogramming by 
baicalin is functional in regulating cytokine production. Overall, baicalin promoted the TAM repolarisation towards M1-like phenotype.
Baicalin induces repolarisation of M2-like macrophage without affecting M1 phenotype. To further examine the action of baicalin on repolarisation of differentiated macro-
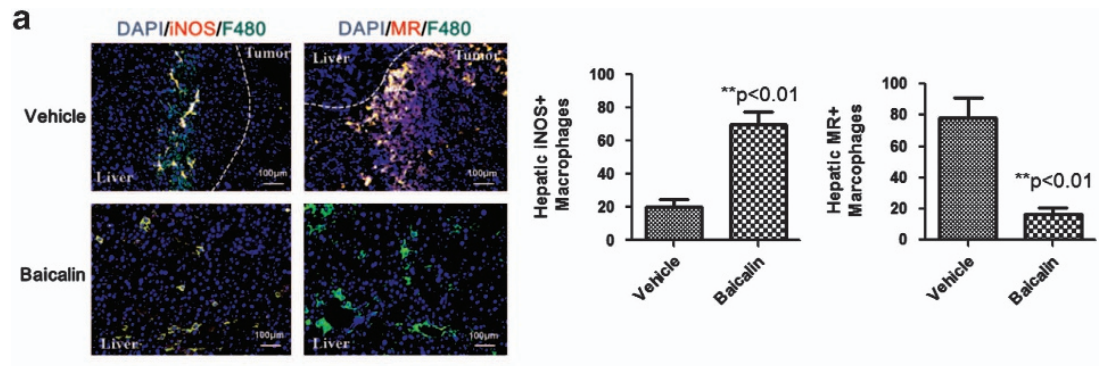

b
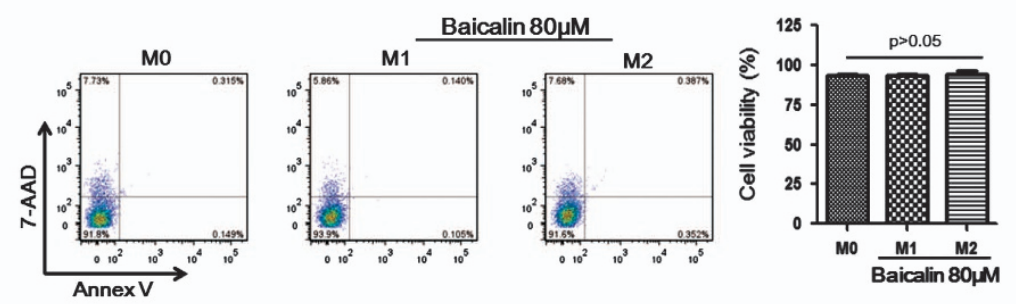

c

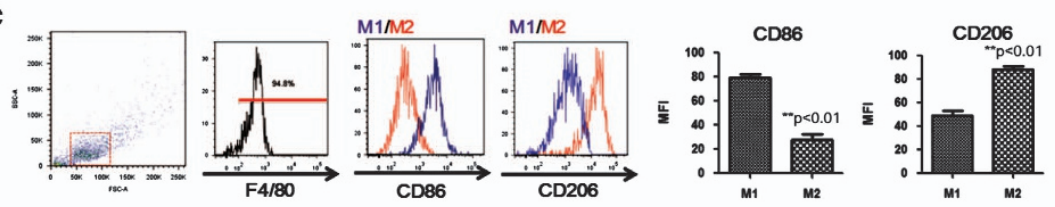

d
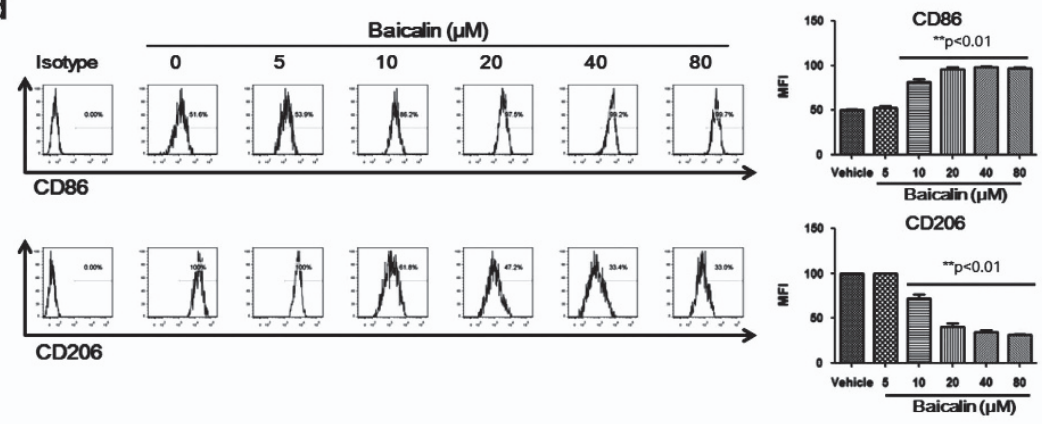

e

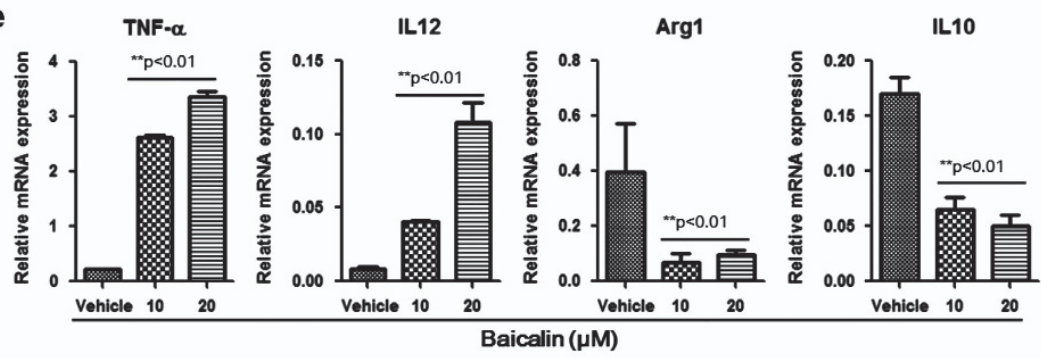

f

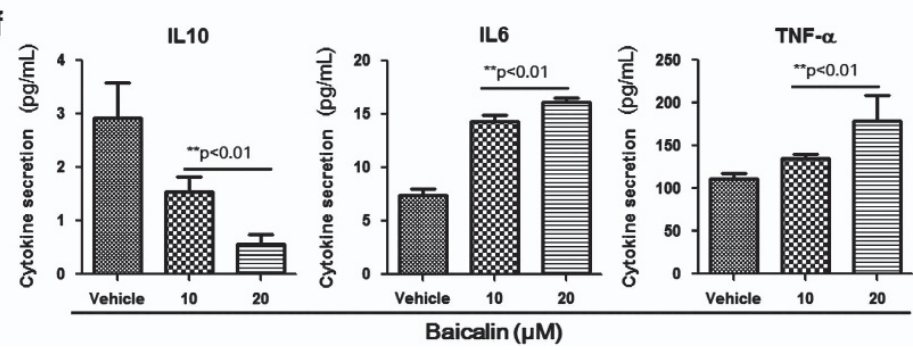


phages, we polarised BMDMs-derived macrophages to either M1-like or M2-like phenotype. The polarised macrophages were then subject to $48 \mathrm{~h}$ treatment of baicalin. Interestingly, baicalin particularly skewed M2-like macrophages towards M1-like phenotype, without causing any significant phenotypic changes in M1-like macrophage (Figure 3a). This was further evidenced by the fact that expression of proinflammatory genes in baicalin-treated M2-like macrophages was increased while anti-inflammatory factors were reduced (Figure 3b). In M1-like macrophages, expression of proinflammatory genes also increased though no phenotypic changes were observed after baicalin treatment, indicating that baicalin treatment could promote pro-inflammatory cytokines production. Consistent observation was obtained in culture supernatant of both M1-like and M2-like macrophages with baicalin treatment (Figure 3c). As M1-like TAMs may exert their anti-tumour action by producing pro-inflammatory cytokines, ${ }^{40}$ these observations revealed that baicalin treatment may result in polarised macrophages in tumour microenvironment prone to acquire anti-tumour functions. And the effect of baicalin on TAMs repolarisation in tumour microenvironment may be independent to any changes in polarisation factors derived by tumour cells itself, as phenotypic analysis showed that TAMs cultured with TSN from HCC cells with pre-treatment of baicalin were not prone to M1-like phenotype (Figure 3d). All the above observations confirmed the direct reprogramming action of baicalin on polarised TAMs. Furthermore, co-culturing of baicalin-pretreated TAMs with HCC cells suppressed motility of cancer cells. HCC cells were seeded with $100 \%$ confluence in 24-well cell culture plate and a gap was created by scraping with a $10-\mu l$ pipette tip at the centre of the monolayer. Then HCC cells were subject to $48 \mathrm{~h}$ co-culturing with TAMs pre-treated with vehicle or $20 \mu \mathrm{M}$ baicalin for $48 \mathrm{~h}$. HCC cells co-cultured with baicalin-pre-treated TAMs exhibited slow migration towards centre of the gap (Figure $3 e, P<0.01$ ). Also, co-culturing of baicalin-pre-treated TAMs inhibited proliferation of HCC cells. HCC cells $\left(1 \times 10^{4}\right)$ were seeded and were subject to a co-culturing with TAMs pre-treated with vehicle or $20 \mu \mathrm{M}$ baicalin. Number of HCC cells was counted at day 3, 6 and 9. Reduced proliferation of HCC cells was observed in time-dependent manner (Figure $3 f, P<0.01$ ). Our results indicate that baicalin suppressed tumour cell expansion by incurring direct reprogramming effect on polarised macrophages.
Induction of autophagy is involved in repolarisation of baicalin-treated TAMs. Some previous studies have reported the property of baicalin as a natural autophagy inducer in cancer cells, ${ }^{34,41}$ and consistently, we observed increased of LC3-II/LC3-I ratio in baicalin-treated TAMs. The conversion of cytoplasmic form of LC3 into membrane form in baicalin-treated TAMs indicated induction of autophagy (Figure 4a). We also observed autophagy induction in TAMs on liver tissue of HCC bearing mice (Supplementary Figure 7). Elevated LC3 punctuation in baicalin-treated TAMs further proved the autophagy initiation, and baicalin-induced autophagy could be blocked by RNA interference against autophagy essential gene Atg5 (Figure 4b). It was found that induction of autophagy by baicalin could only be achieved in unpolarised and M2-like macrophages but not in M1-like macrophages (Figure 4c). Considering that, ${ }^{42}$ this finding may indicate autophagy plays a role in baicalin-induced macrophage polarisation. To prove this hypothesis, we used Bafilomycin A1 and RNA interference against Atg5 gene to suppress induction of autophagy in TAMs. Pharmacological inhibition of autophagy by Bafilomycin A1 abolished repolarisation of TAM by baicalin towards M1-like phenotype (Figure 4d), and it was further confirmed by reduced pro-inflammatory genes and elevated anti-inflammatory genes in baicalin-treated TAMs in the presence of Bafilomycin A1 (Figure 4e). Consistent observations was obtained on reprogramming of baicalin-treated TAMs towards M2-like phenotype upon Atg5 depletion, and reverted changes of relative gene expression induced by baicalin (Figures $4 \mathrm{f}$ and g). These observations confirmed autophagy is involved in reprogramming of TAM induced by baicalin.

\section{Autophagy-associated activation of RelB/p52 pathway mediates repolarisation of TAM by baicalin. To explore the mechanism underlying autophagy-induced repolarisation of TAM by baicalin, we examined the activation of RelB/p52 pathway, which was reported to dominate reprogramming of Th1 cells to Th2 phenotype. ${ }^{43}$ Increased RelB expression was observed in baicalin-treated TAMs, which could be attenuated by depletion of Atg5 (Figure 5a). Interestingly, it was observed that in baicalin-treated TAMs, the mRNA expression of RelB was elevated (Supplementary Figure S2). The consistent increase of phosphorylation of p100 and p52 in baicalin-treated TAMs revealed that the transcriptional increase of RelB may be due to the positive feedback loop of}

Figure 2 Treatment of baicalin results in reprogramming of M2-like TAMs to M1-like phenotype. (a) Baicalin treatment resulted in increase of hepatic M1-like TAMs with reduced M2-like populations. Antibodies against iNOS (M1-like marker) and mannose receptor (MR, M2-like marker) were used to stain frozen-sectioned liver tissue. Increase of iNOS-positive cells all over the liver of baicalin-treated mice was observed $(P<0.01)$; while hepatic MR-positive cells within non-tumour and peri-tumour area was remarkably reduced upon baicalin treatment $(P<0.01)$ (scale bar, $100 \mu \mathrm{m}$ ); (b) baicalin did not cause cell death in any specific type of macrophages. BMDM was cultured and differentiated as described in Materials and Methods. A relatively high dose of baicalin $(80 \mu \mathrm{M})$ was given to either M1-like or M2-like TAM for $48 \mathrm{~h}$. Cell viability was determined with flow cytometry using Annexin V/7-AAD staining. (c) Differentiated BMDM exhibited differential patterns in CD86/CD206 expression. Antibodies against CD86 and CD206 were used to stain cell surface marker of differentiated BMDMs. F4/80 was co-stained to identify differentiated macrophage population. M1-like macrophage had higher expression of CD86 with lower expression of CD206 $(P<0.01)$, while CD206 was induced and CD86 was relatively low in M2-like macrophage $(P<0.01)$. (d) Baicalin treatment resulted in skewing of TAM to M1-like macrophage. TAMs were induced by culturing BMDM with tumour supernatant (TSN) as described in Materials and Methods. Cells were subjected to different doses of baicalin for $48 \mathrm{~h}$. It was observed that baicalin induced skewing of TAMs into M1-like phenotype and away from M2-like in dose-dependent manner. (e) Cytokine profile in baicalin-treated TAMs favouring M1-like phenotype. RNA from baicalin-treated cells was collected and expression of TNF- $\alpha$, IL12 (M1-like macrophage markers) and IL10, Arginase1 (Arg1, M2-like macrophage markers) was determined by quantitative real-time PCR. Increase of M1 markers but decrease of M2 markers was observed in baicalintreated TAM $(P<0.01)$. (f) Baicalin treatment induced elevated secretion of pro-inflammatory cytokine in TAMs. Cytokine concentration in cultured TAM supernatant was determined with CBA kit. TNF- $\alpha$ and IL6 (pro-inflammatory cytokines) levels were increased $(P<0.01$ ), while IL10 (anti-inflammatory cytokine) was reduced after baicalin intervention $(P<0.01)$. Cytokine level was expressed as $\mathrm{pg} / \mathrm{ml} \pm$ S.D. 
a
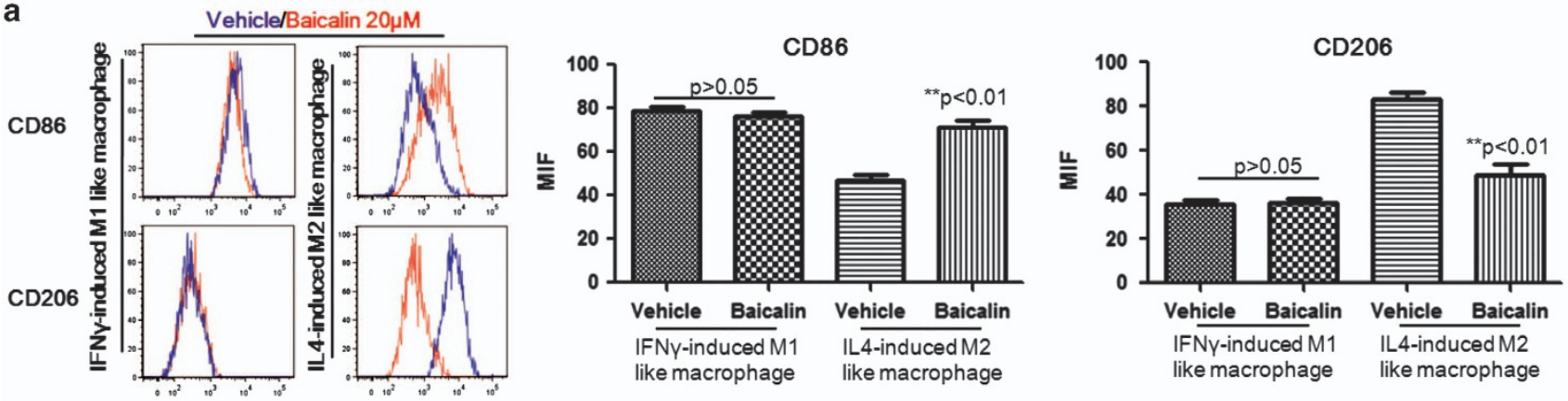

b
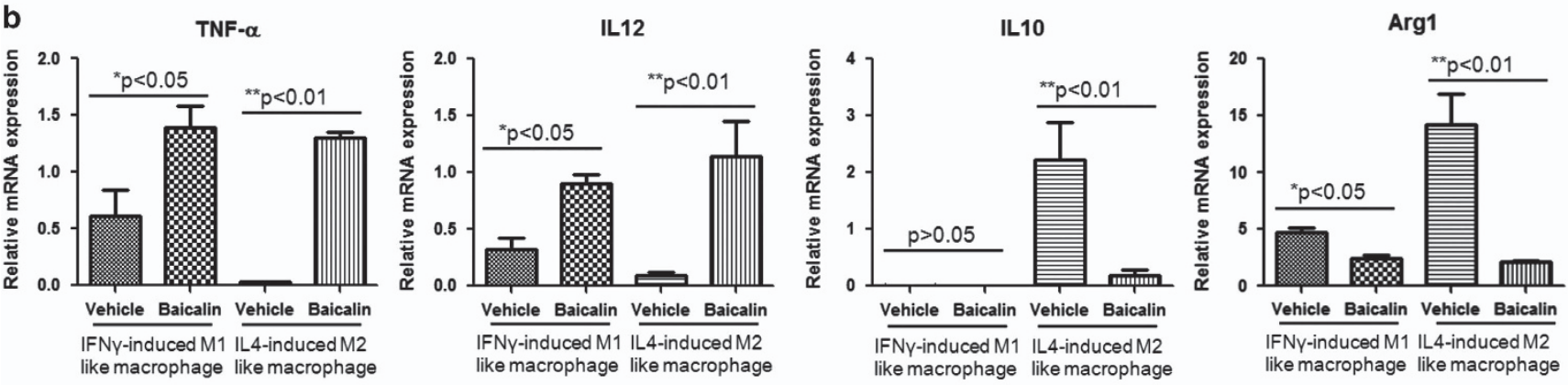

C

IL10

IL6
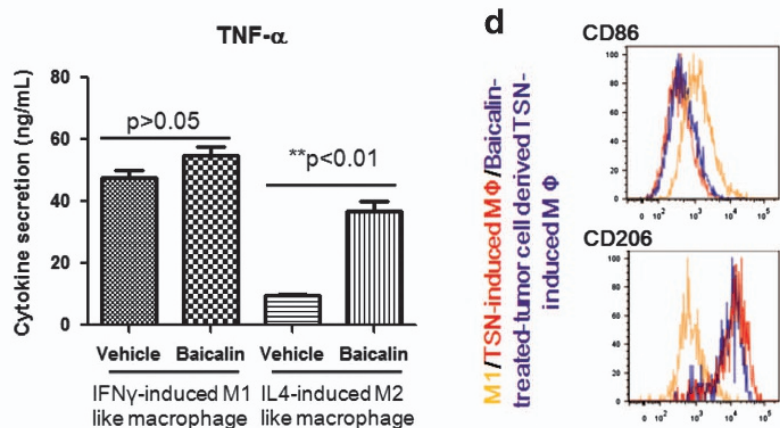

CD206

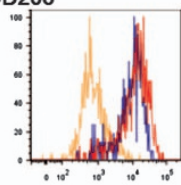

e
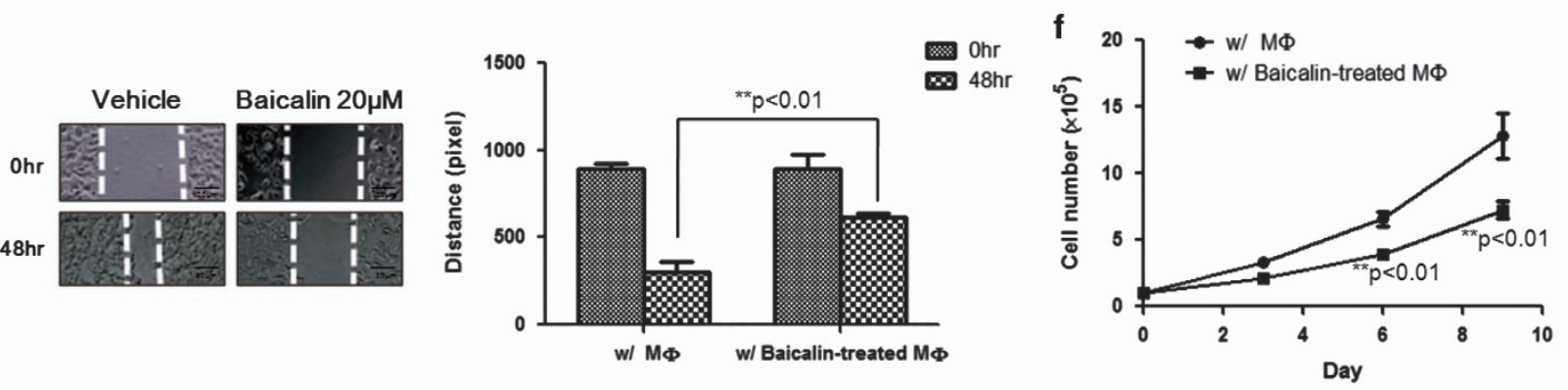

Figure 3 Baicalin induces repolarisation of M2-like macrophage without affecting M1 phenotype. (a) The $20 \mu \mathrm{M}$ baicalin treatment repolarised differentiated M2-like macrophage to M1 phenotype, while minimally affected differentiated M1-like macrophage. BMDMs were cultured and differentiated, then treated with baicalin for $48 \mathrm{~h}$. Expression of CD86/CD206 was determined $(P<0.01)$. Increase of CD86 expression with CD206 suppression was found in baicalin-treated M2-like macrophage, while baicalin had minimal effect on the expression of cell surface markers in M1-like macrophage $(P>0.05)$. This observation was further evidenced by cytokine expression profile (b) and cytokine secretion (c). (d) Baicalin had minimal effect on macrophage-polarisation factors derived by tumour cells. TSN was collected from HCC cells treated with either vehicle or $20 \mu \mathrm{M}$ baicalin, and was used to differentiate BMDMs as described in Materials and Methods. No significant skewing of TAM could be observed. Differentiated M1 macrophages were used as a positive control. (e) Co-culturing of baicalin-pre-treated TAMs suppressed motility of $\mathrm{HCC}$ cells. HCC cells were seeded in 24-well cell culture plate and a gap was created by scraping with a $10-\mu$ l pipette tip at the centre of the monolayer. Then HCC cells were subject to a 48-h co-culturing with TAM pre-treated with vehicle or $20 \mu \mathrm{M}$ baicalin for $48 \mathrm{~h}$. HCC cells co-cultured with baicalin-pre-treated TAM exhibited slow migration towards centre of the gap $(P<0.01)$. (f) Co-culturing of baicalin-pre-treated TAM inhibited proliferation of $\mathrm{HCC}$ cells. $\mathrm{HCC}$ cells $\left(1 \times 10^{4}\right)$ were seeded and subjected to co-culturing with TAMs pre-treated with vehicle or $20 \mu \mathrm{M}$ baicalin. Number of HCC cells was counted at day 3, 6 and 9. Reduced proliferation of HCC cells with baicalin-pre-treated TAM was observed in time manner $(P<0.01)$ 
RelB/p52 activation. ${ }^{44}$ Co-immunoprecipitation assay with RelB antibody confirmed association of p52 with RelB in TAMs, while $\mathrm{I}_{\kappa} \mathrm{B} a$ was dissociated from the RelB/p52 complex with baicalin treatment (Figure 5b). Furthermore, it was observed that RelB and p52 were translocated into nuclear area of TAMs in the presence of baicalin (Figure $5 \mathrm{c}$ ), and transactivated the RelB/p52-specific target genes CCL9 and CXCL12 (Figure $5 d$ ). Inhibition of RelB expression by RNA interference in baicalin-treated TAMs abolished repolarisation of TAMs towards M1-like phenotype, and drove the tendency of TAMs towards M2-like phenotype. Increased expression of pro-inflammatory cytokines by baicalin was abolished by RelB suppression, and reduction of anti-inflammatory cytokines was restored (Figure $5 f$ ). The high expression of RelB and 052 in M1-like macrophages instead of in unpolarised and M2-like macrophages (Figure5g) further confirmed the role of upregulated ReIB in mediating repolarisation of TAM induced by baicalin.

Activation of RelB/p52 pathway may be dependent on reduced expression of TRAF2 in baicalin-treated TAMs. To further prove the mechanism, we observed the expression of IKKa, the upstream event of RelB/p52. A consistent upregulation of IKKa was observed in baicalin-treated TAMs, which was blocked by inhibition of Atg5 (Figure 6a). Similar to RelB, it was found that expression of IKKa mRNA was elevated (Supplementary Figure S3), majorly due to the positive feedback regulation of RelB/p52 activation. ${ }^{45}$ The role of IKKa in mediating baicalin-induced RelB activation was further proved by the observation that IKKa inhibition by RNA interference attenuated upregulation of RelB expression (Figure 6b). Interestingly, it was shown that blockade of IKKa has minimal effect on baicalin-induced autophagy in TAM, indicating that IKKa positions at the downstream of autophagy (Figure 6c). Silencing of IKKa in baicalin-treated TAMs restored M2-like phenotypic characteristics of the cells (Figure 6d), and suppressed pro-inflammatory gene expression in baicalin-treated TAMs, as well as restored the expression of anti-inflammatory genes (Figure 6e). As IKKa also plays a key role in mediating activation of RelA by inducing phosphorylated-degradation of $\mathrm{I}_{\kappa} \mathrm{Ba},{ }^{46}$ which then activate transcription of pro-inflammatory genes, we examined if RelA pathway was affected by baicalin treatment in TAMs. Interestingly, we observed very low expression level of RelA in TAMs, and baicalin treatment could suppress RelA even in the presence of soluble TNF-a, a RelA-activating cytokine (Figure 6f). This was consistent with the previous report that TLR2 could selectively suppress RelA expression upon induction of autophagy. ${ }^{47}$ This data revealed that repolarisation of TAM by macrophage may be dependent on activation of RelB but not RelA pathway. In this case, we then examined the expression of TRAF2/TRAF3 complex, the negative regulator of RelB pathway, and observed the reduced expressions of both TRAF2 and TRAF3 (Figure $6 \mathrm{~g}$ ). Our data may suggest restriction of TRAF2/TRAF3 expression, the intracellular suppressor of IKKa/ReIB/p52 signalling, may confer repolarisation of TAM by baicalin.

Baicalin initiates autophagic degradation of TRAF2 in TAMs. In our study, we did not observe reduced mRNA expression of TRAF2 in baicalin-treated TAM (Supplementary Figure S4), though protein level of TRAF2 was potently suppressed (Figure $6 \mathrm{~g}$ ). This inhibitory effect of baicalin on TRAF2 was completely abolished in the presence of pharmacological inhibitor of autophagy Bafilomycin A1, or by depleting Atg5 from TAMs (Figure 6h), indicating that reduced expression of TRAF2 in baicalin-treated TAMs may be independent to transcriptional suppression of TRAF2 itself but dependent on autophagic degradation pathway. And this effect of baicalin was specific to M2-like macrophages, as expression of TRAF2 was relatively low in M1-like macrophages and baicalin showed minimal effect on it (Figure 6i). This selective autophagic degradation of TRAF2 in baicalin-treated TAMs was further proved by observation of co-localization of TRAF2 with lysosome and monodansylcadaverine (MDC), indicating a lysosomal degeneration of TRAF2 induced by baicalin (Figure 6j and Supplementary Figure S6). Co-immunoprecipitation assay with LC3 and TRAF2 antibody also confirmed the association of TRAF2 with LC3 and Atg5 in TAMs after baicalin intervention (Supplementary Figure S6). Furthermore, a cargo protein of selectively autophagic degradation, p62, was observed to attach TRAF2 upon baicalin treatment (Figure 6k). These observations reveal a lysosomal degradation of TRAF2 is dependent on baicalin-induced autophagy in TAMs.

\section{Discussions}

Macrophages are high-plasticity immune cells, and they maintain in M1/M2 mixture states under normal condition. Macrophage M1/M2 state switching may be crucial in determining the tissue fate. ${ }^{48}$ High expression of M-CSF and macrophages markers in peritumoral area of hepatocellular carcinoma tissue promotes tumour recurrence and metastasis; distribution of M2 macrophages in peritumoral section is associated with poor prognosis of HCC patients. ${ }^{3,49}$ Depletion of TAMs further improves the anti-tumour effect of sorafenib, the current first-line treatment of HCC. ${ }^{50}$ Therapeutics against M2 macrophage is no doubt, in most of the studies, benefits cancer therapy. However, the role of M1like macrophage is much more complicated as previous studies showed that the inflammatory environment conditioned by macrophages promoted tumorigenesis and cancer progression. It was shown that IL6-producing TAM may promote the expansion of cancer stem cell in $\mathrm{HCC} .{ }^{51}$ Although phenotype of TAM cannot be identified here, as both M1-like and M2-like macrophages could produce IL6, ${ }^{52}$ it indicates the role of pro-inflammatory M1 macrophages may not be as direct as M2 macrophages. A recent study observed that though TAM was repolarised towards M1 phenotype and away from M2 phenotype by sorafenib, the circulating inflammation condition was reduced by the treatment. ${ }^{4}$ This may indicate that skewing of M2-like TAM to M1 phenotype will not promote systematic inflammation in cancer patients. In fact, HCC carcinogenesis would request infiltration of inflammatory leukocytes into the liver, which may cause hepatocyte apoptosis and sustained inflammation. ${ }^{53}$ Our study, showing that baicalin did not elevate circulating inflammatory monocytes, revealed that the compound may be able to repolarise loco-regional TAM away from M2 phenotypes. In fact, in our co-culture study, we did 
not observe significant apoptosis of $\mathrm{HCC}$ cells in the presence of baicalin-pre-treated TAMs (data not shown), which may indicate that production of pro-apoptotic factors by baicalin-treated
TAMs, such as TNF- $a$, is not sufficient to cause death of HCC cells. Our findings, though baicalin-treated TAMs are prone to be M1-like, we cannot preclude that reduced HCC cell
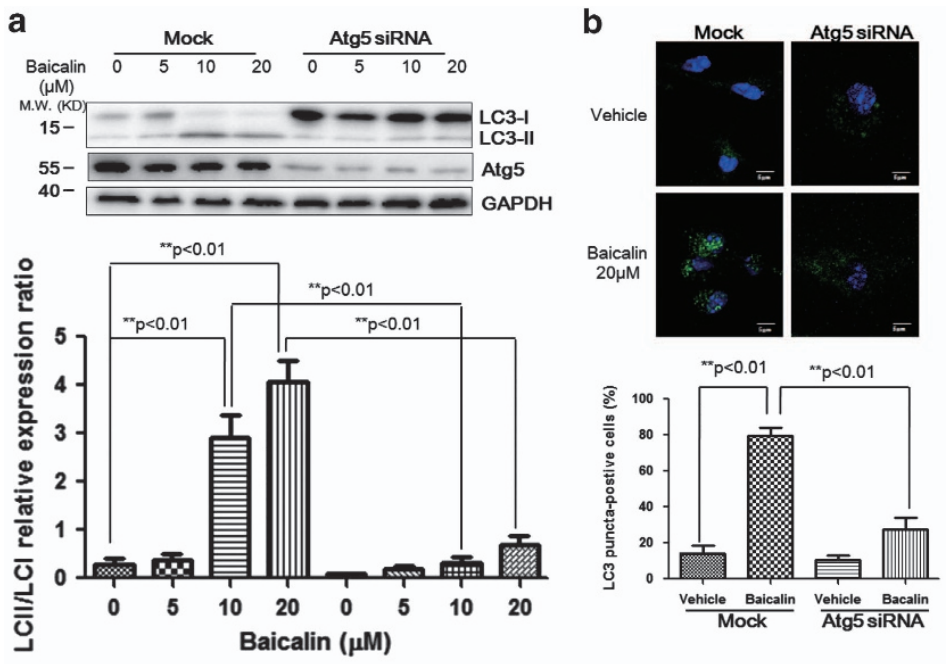

c
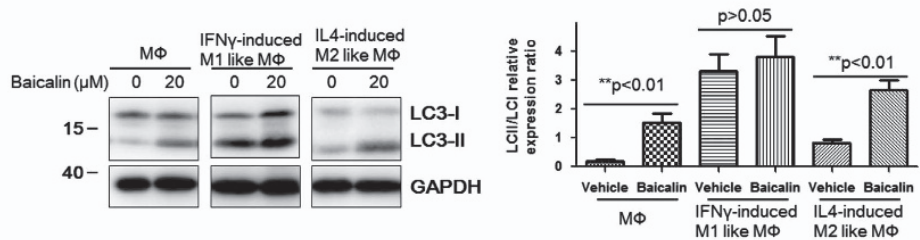

d
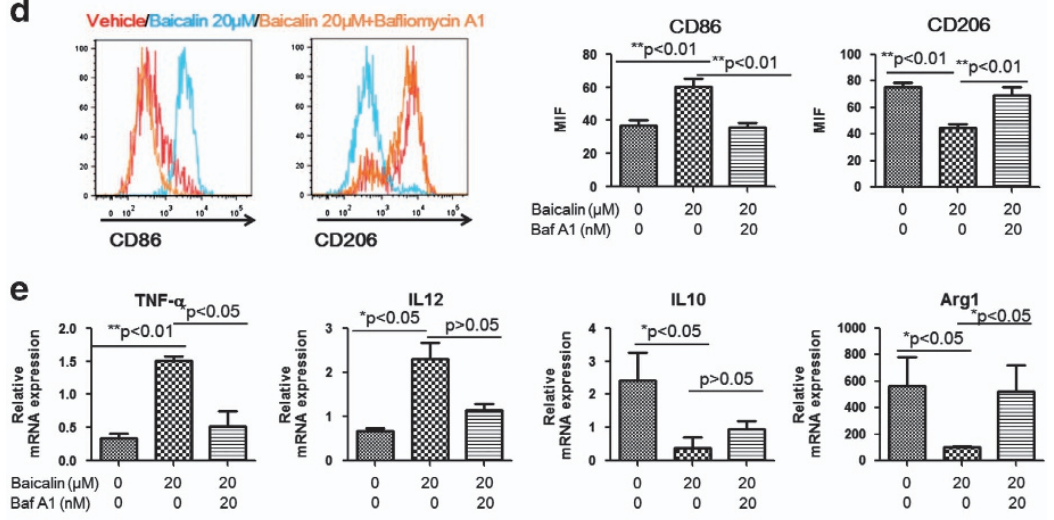

f
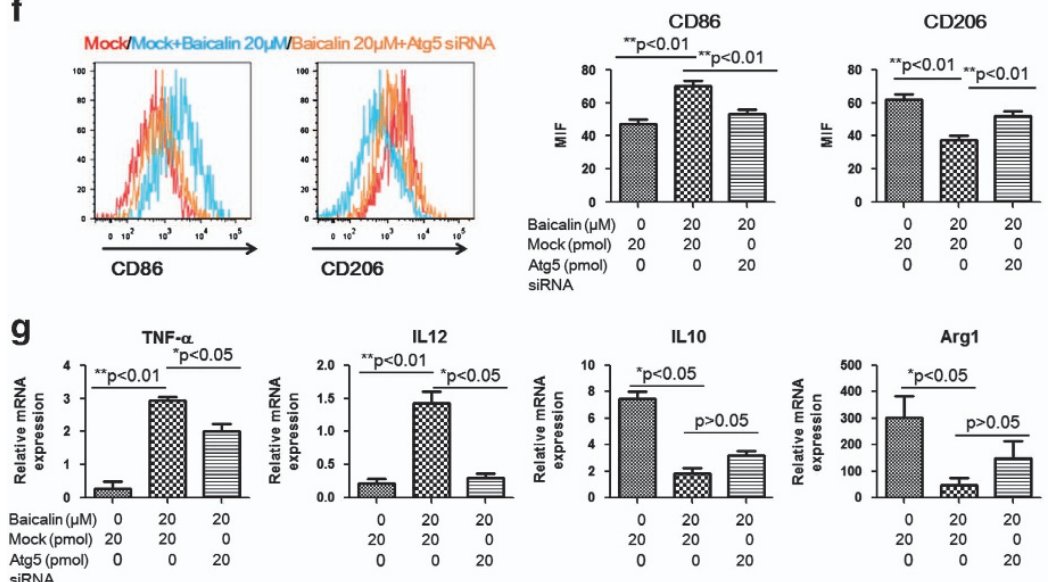
proliferation and migration is not due to the decrease of M2-like TAM-derived protumoral cytokines.

Furthermore, our study demonstrated that baicalin-mediated M1 reprogramming is regulated by the downregulation of TRAF2 via autophagy-dependent pathway. Detailed regulatory mechanism is illustrated in Figure 7. TRAF2 is a signalling protein in response to TNF receptor binding activity, and its regulation largely affected the downstream NF- $\kappa$ B activation. In TNF-RII complex, association of TRAF2 with c-IAP1 promotes TRAF2 ubiquitination-dependent degradation. ${ }^{54}$ The c-IAP1-mediated TRAF2 degradation is required for monocyte differentiation to functional macrophages. ${ }^{55}$ It is

a

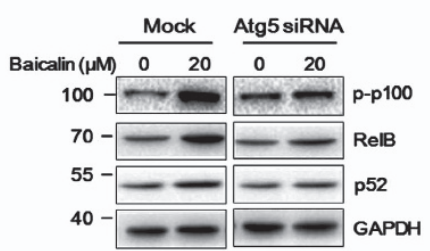

e

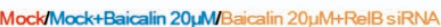
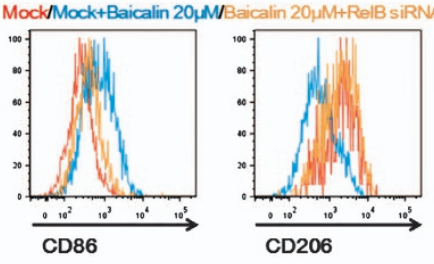

b
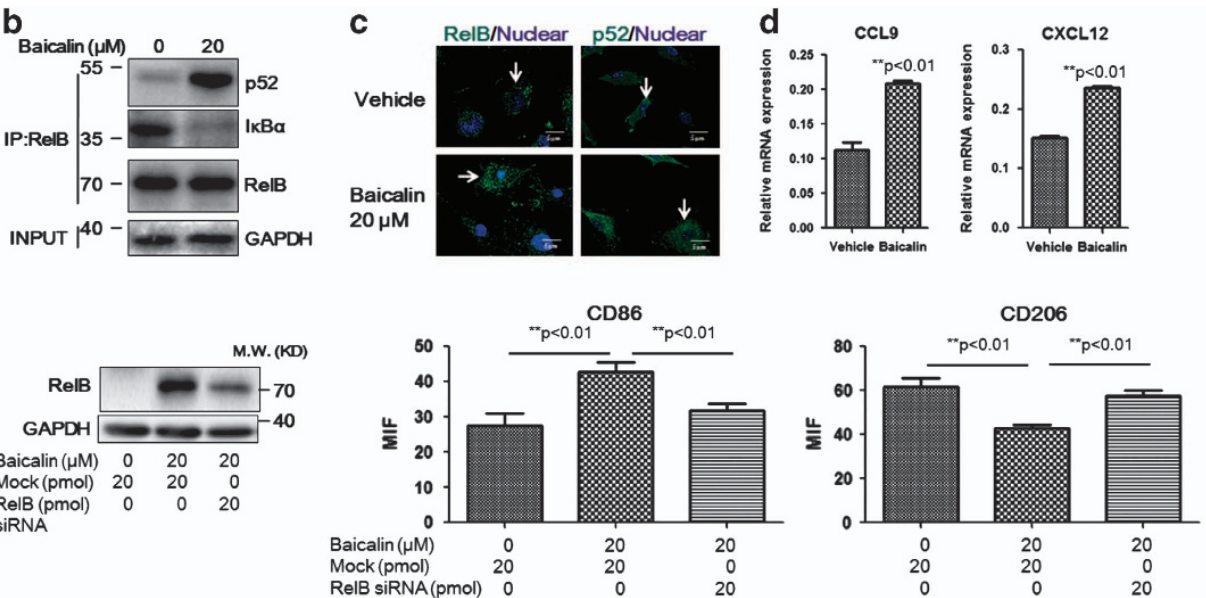

$\mathbf{f}$
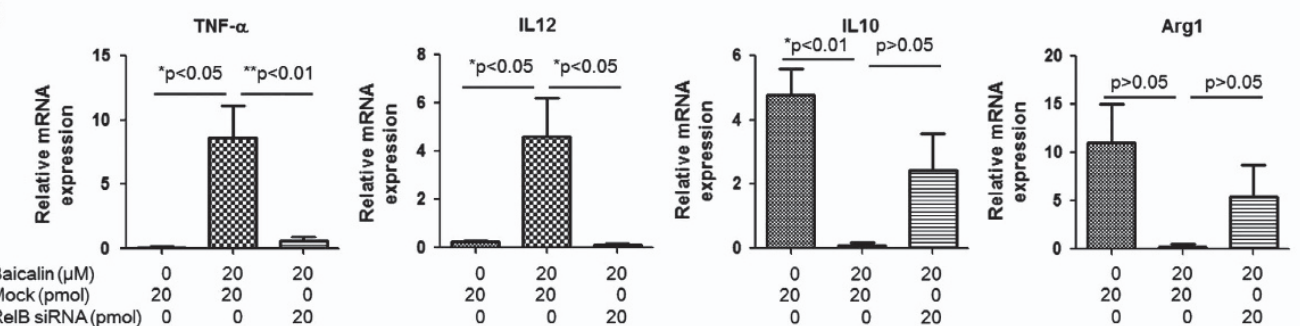

g
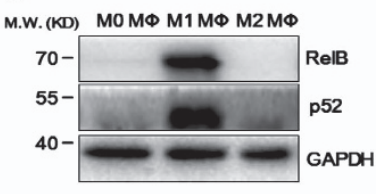

RelB siRNA(pmol) 0

Figure 5 Autophagy-associated activation of RelB/p52 pathway mediates TAM repolarisation induced by baicalin. (a) The induced expression of RelB in baicalin-treated TAM is associated with autophagy induction. RNA interference against Atg5 was conducted as described and TAM was treated with $20 \mu \mathrm{M}$ baicalin for $48 \mathrm{~h}$. Expression of RelB, $\mathrm{p}$-p100 and p52 was detected by immunoblotting. It was shown that baicalin treatment resulted in increase of RelB and p52 expression, as well as phosphorylation of p100, and this effect of baicalin could be blocked by inhibition of autophagy by RNA interference against Atg5. (b) Baicalin treatment induced association of p52 with RelB. TAM was treated and coimmunoprecipitation assay was conducted as described. Increased association of RelB with p52 was observed in baicalin-treated TAM. Reduced IkB $\alpha$ with RelB was also observed. Expression of protein level was normalised by GAPDH as INPUT samples and by RelB as IPed-samples. (c) Baicalin treatment induced nuclear localisation of RelB and p52. DAPI was used to stain nuclei of the cells. TAM with or without baicalin treatment was fixed and stained with antibodies against RelB and p52, followed by observation under confocal microscope. Increased RelB and p52 presentation in nuclear area was found in baicalin-treated cells. (d) Elevation of RelB/p52-specific target gene expression CCL19 and CXCL12 was observed in TAM in the presence of baicalin (magnification: x60). (e) Suppression of RelB by RNA interference induced re-skewing of baicalin-treated TAMs to M2 phenotype. RNA interference was conducted as described and TAM was subjected to baicalin treatment. Suppression of RelB expression significantly blocked change of CD86 and CD206 expression induced by baicalin. This was further evidenced by observation in cytokine expression profile (f) of baicalin-treated TAMs with or without RNA interference against RelB. (g) Expression of RelB and p52 in different phenotypes of macrophages. BMDMs were cultured and differentiated as described and protein was collected for analysis. It was observed that RelB and p52 were highly expressed in M1-like macrophage but not in either unpolarised or M2-like macrophage

Figure 4 Autophagy is involved in baicalin-induced TAM repolarisation. (a) Baicalin treatment induced conversion of LC3-I into LC-II in TAM. BMDM cells were subjected to RNA interference as described in Materials and Methods with mock scramble negative control or siRNA against murine Atg5. Cells were treated with baicalin for $48 \mathrm{~h}$ and protein was collected for analysis. Increase of LC3-II expression with reduced LC3-I upon baicalin treatment was observed, while suppression of Atg5 attenuated the conversion of LC3-I into LC-II. (b) Baicalin increased LC3 puncta in TAM. Cells with mock or Atg5 RNA interference were subjected to baicalin treatment. Then cells were stained with LC3B antibody and visualised under confocal microscope. Increase of LC3 puncta (light green dots) was observed in baicalin-treated TAM, which could be blocked by RNA interference against Atg5 (magnification: x60). (c) Baicalin could induce autophagy in M2-like macrophage but not in M1-like. Protein expression was detected with immunoblotting. Conversion of LC3-I into LC3-II was observed in both unpolarised macrophage and M2-like macrophage, but not in M1-like macrophage. (d) Blockade of autophagy by presence of Bafilomycin A1 resulted in re-skewing of baicalin-treated TAMs into M2 phenotype. Bafilomycin A1 was added to TAM 30 min before the 48-h baicalin treatment. It was observed that presence of Bafilomycin A1 potently blocked change of CD86 and CD206 expression induced by baicalin. This was further evidenced by observations in cytokine expression profile (e) of baicalin-treated TAMs with or without Bafilomycin A1. (f) Suppression of autophagy by RNA interference against Atg5 induced re-skewing of baicalin-treated TAM to M2 phenotype. RNA interference was conducted as described and TAMs was subject to baicalin treatment. Suppression of Atg5 expression significantly blocked change of CD86 and CD206 expressions induced by baicalin. This was further evidenced by observations in cytokine expression profile (g) of baicalin-treated TAM with or without RNA interference against Atg5 
surprising that we found the blockade of autophagy also inhibited TRAF2 reduction, suggesting TRAF2 may be degraded in lysosome apart from the conventional proteasomal-related pathway. Although this scenario may be rare; the notion is supported by a recent study on TRAF2 lysosomal-dependent degradation mediated by zinc finger
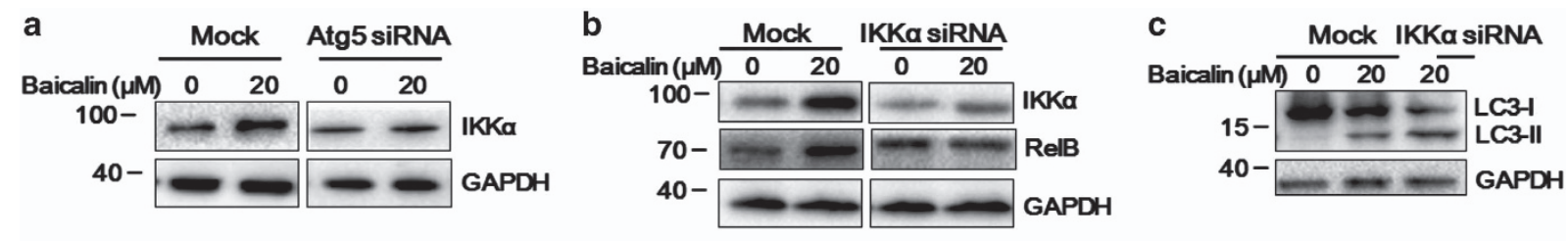

d
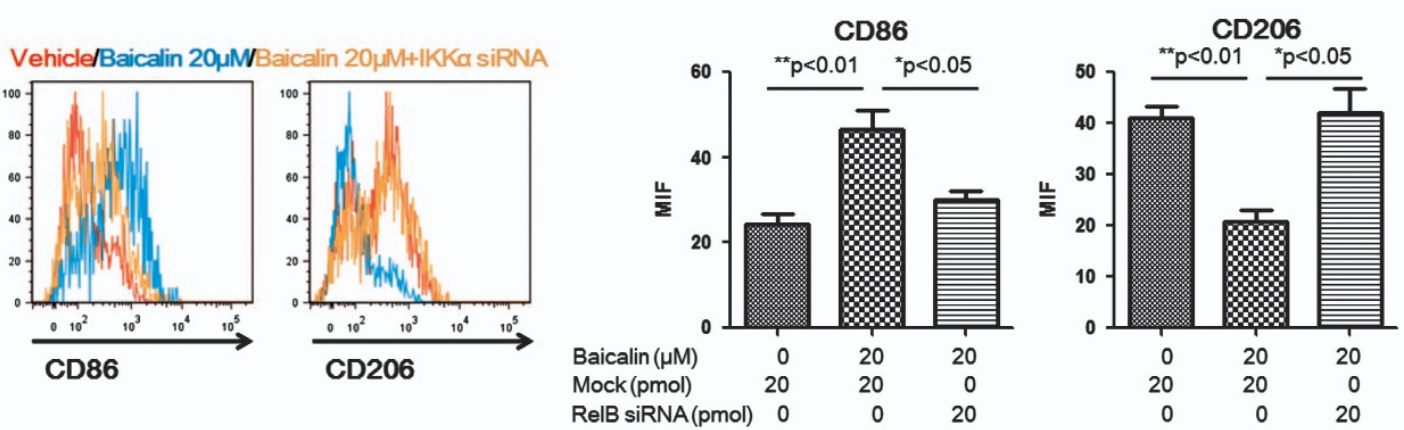

e
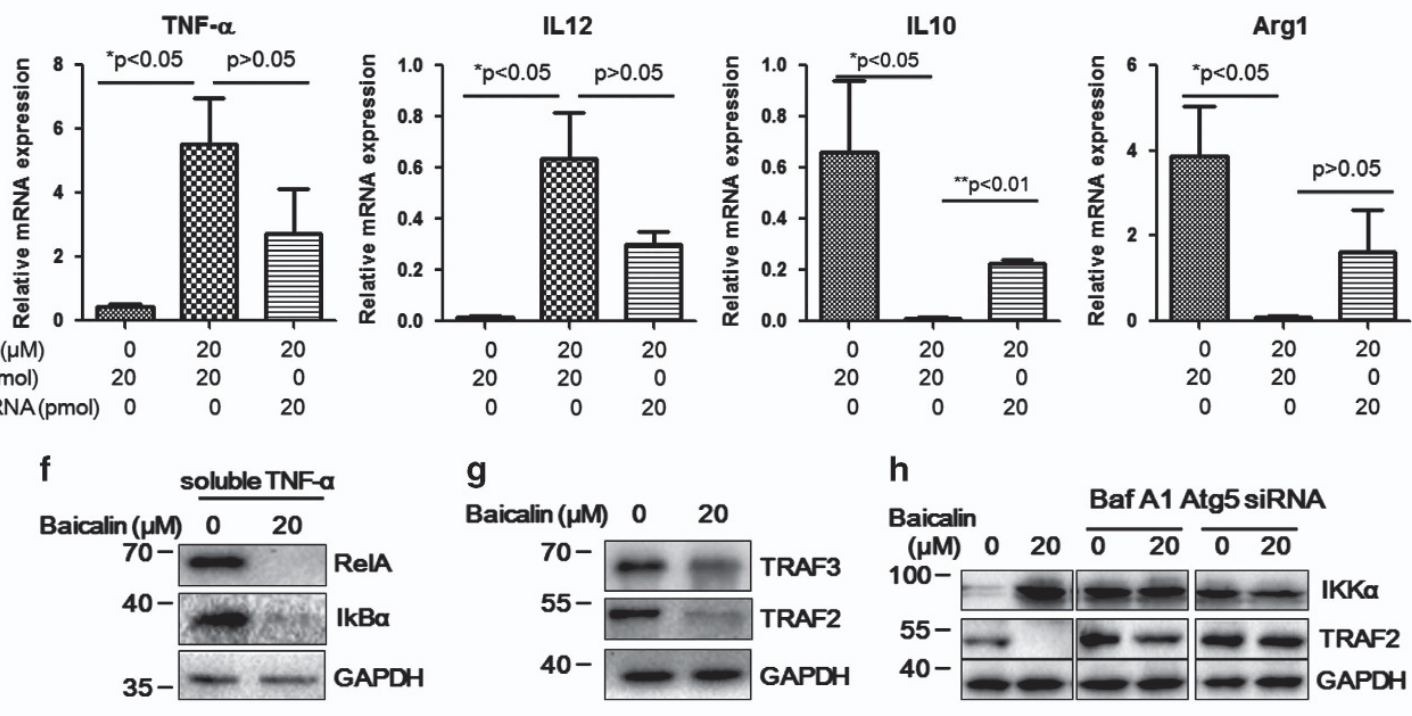

g

Baicalin $(\mu \mathrm{M}) \quad 0 \quad 20$

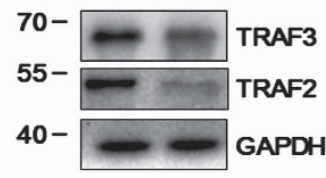

h
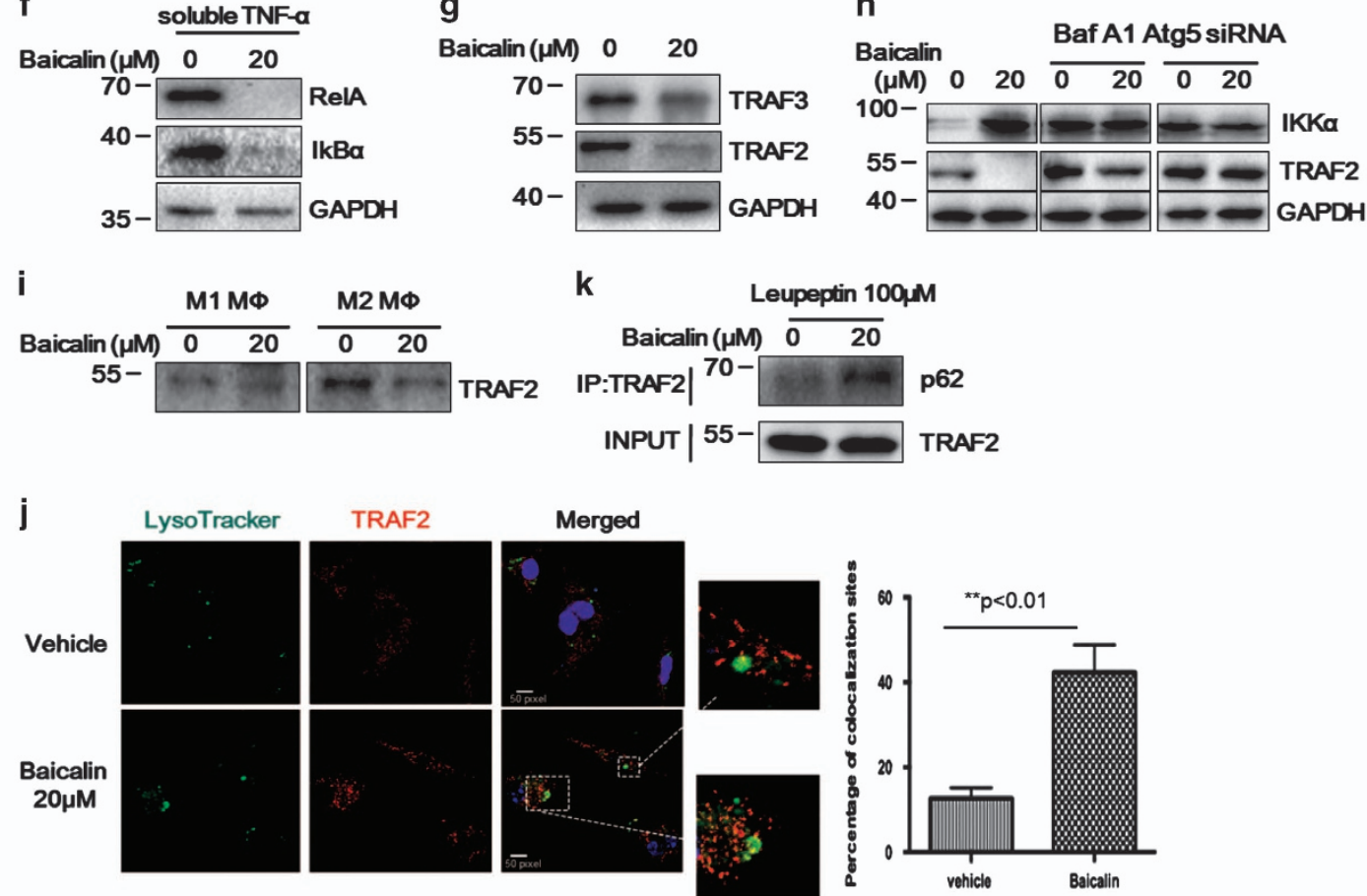
protein $\mathrm{A} 20 .^{56}$ We indeed observed the ubiquitination of TRAF2 (Supplementary Figure S5) and colocalization of TRAF2 with lysosomal tracker p62, the autophagy-related protein that cargo ubiquitinated intracellular molecules, which was recruited to autophagosome during autophagy process and lead to the targeted molecules degraded through lysosomal pathway. ${ }^{57}$ The interaction between ubiquitinated TRAF2 and p62 may explain TRAF2-lysosomal degradation is autophagy dependent. All these collectively suggest the recruitment of ubiquitinated TRAF2 to lysosomal compartments for clearance.

In our study, baicalin-mediated downregulation of TRAF2 promotes sustained activation of IKKa and RelB. IKKa is a tumour promoter in the context of colorectal cancer that is responsible in blocking the recruitment of M1-like myeloid cells. ${ }^{58} \mathrm{IKKa}$ and RelB are important regulators of noncanonical NF- $\kappa \mathrm{B}$ signalling and $\mathrm{p} 100$ processing contributes to the activation of RelB/p52 complex, while TRAF2 negatively regulates the pathway. ${ }^{59}$ Inactive form of RelB is associated with $\mathrm{I}_{\kappa} \mathrm{B} a$ and it mobilises to nucleus followed by $\mathrm{I}_{\kappa} \mathrm{B} a$ degradation. ${ }^{60}$ RelB is associated with p52 to activate transcription of target genes. This non-canonical activation of $\mathrm{NF}-\kappa \mathrm{B}$ pathway has been reported to dominate the reprogramming of Th1 cells into Th2 phenotype in cells other
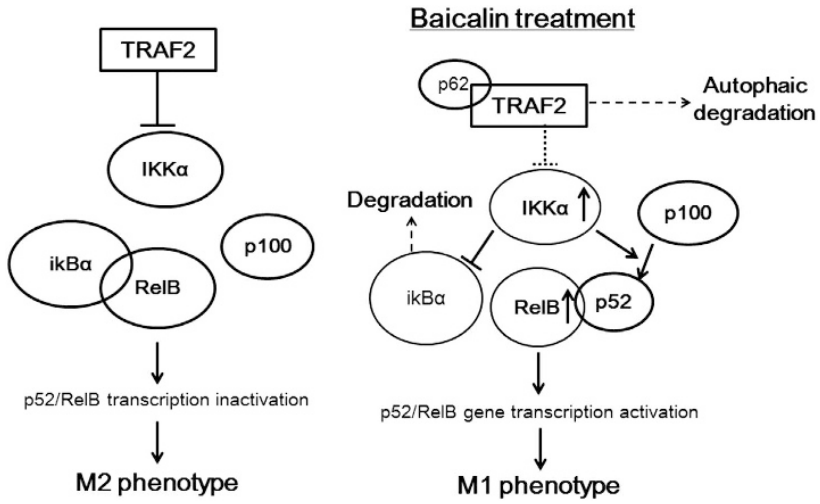

Figure 7 The mechanism underlying repolarisation of baicalin-treated TAMs to M1 phenotype than macrophage. ${ }^{43}$ Besides, study has showed high nuclear p50 concentration in TAMs associated with M2-like phenotype, while TAMs isolated from p50-knockout mice expressed M1-like cytokines. ${ }^{21}$ The inhibitory effect of p50 on M1-reprogramming may be due to the suppression of NF- $\kappa \mathrm{B}$ transcriptional activities through formation of p50 homodimers. ${ }^{61}$ As p50 lacks of transactivation domain, p50/p50 homodimer is supposed to compete with p65/p50 heterodimer, which is able to activate transcription of proinflammatory genes. ${ }^{11}$ In fact, p52, the non-canonical NF- $\kappa B$ pathway member, which also lacks of transactivation domain, is able to bind to itself or with $p 50$ to act as a repressor of NF- $k \mathrm{~B}$ activation. ${ }^{62}$ These evidences showed that activation of NF- $\kappa B$ pathway does not merely depend on increased expression of p50 or p52. It was addressed that the binding of NF- $K B$ dimers is highly adaptive to cellular situation. As the transactivation of pro-inflammatory genes by NF- $\kappa \mathrm{B}$ dimers is transient and under dynamic equilibrium, the nature of dimers are dependent on the given cellular concentrations of NF- $k \mathrm{~B}$ components. ${ }^{63}$ In our study, we found that RelB is increased upon baicalin treatment. Increase of RelB may in turn results in conversion of repressive p52 dimers to RelB/p52 heterodimers, which are able to transactive $M 1$ genes expression. This was evidenced by previous study that challenging macrophages with LPS induced RelB expression, which in consequence increased transcription of non-canonical NF- $k$ B-targeted gene ELC. ${ }^{62}$ Also, this was consistent with previous observation that production of RelB is a major contributing factor in activation of non-canonical NF- $k B$ pathways. ${ }^{64}$ Study also revealed that removal of RelA by autophagy, the core molecule of canonical NF- $\kappa$ B pathway, may lead to M2 polarisation of macrophage, ${ }^{47}$ though there is still no direct evidence to show any essential role of RelA pathway activation in mediating macrophage reprogramming. This is consistent with our observation that baicalin-induced autophagy also resulted in RelA degradation in TAM. However, as baicalin-induced autophagy particularly promotes TRAF2 degradation-associated activation of RelB/p52 pathway; the effect of RelA degradation on TAM polarity seems to be compensated. In addition, it was found that production of pro-inflammatory stimuli particularly results in RelB activation but independent to RelA pathway in

Figure 6 Autophagy-induced TRAF2 degradation may be associated with the activation of RelB/p52 pathway in baicalin-treated TAMs. (a) Induced expression of IKK $\alpha$ in baicalin-treated TAMs is associated with autophagy induction. RNA interference against Atg5 was conducted as described and TAM was treated with $20 \mu \mathrm{M}$ baicalin for $48 \mathrm{~h}$. Expression of IKK $\alpha$ was detected by immunoblotting. It was shown that baicalin treatment resulted in increase of IKK $\alpha$ expression, which could be blocked by inhibition of autophagy by RNA interference against Atg5. (b) Silencing of IKK $\alpha$ expression attenuated RelB overexpression in baicalin-treated TAM. RNA interference against IKK $\alpha$ was conducted as described and TAM was treated with $20 \mu \mathrm{M}$ baicalin for $48 \mathrm{~h}$. It was shown that increased expression of RelB by baicalin was attenuated upon IKK $\alpha$ suppression. (c) Autophagy induction is independent to IKK $\alpha$ overexpression. (d) Suppression of IKK $\alpha$ by RNA interference induced re-skewing of baicalin-treated TAM to M2 phenotype. RNA interference was conducted as described and TAM was subjected to baicalin treatment. Suppression of IKK $\alpha$ expression significantly blocked change of CD86 and CD206 expression induced by baicalin. This was further evidenced by observation in cytokine expression profile (e) of baicalin-treated TAMs with or without RNA interference against IKK $\alpha$. (f) RelA pathway may not be activated upon baicalin treatment. TAM was treated with baicalin in the presence of soluble TNF- $\alpha$. Expression of RelA and IKB $\alpha$ was determined. (g) Baicalin treatment induced reduction of TRAF2 and TRAF3 expression. (h) Baicalin-suppressed TRAF2 expression may be blocked when autophagy was blocked by either Bafilomycin A1 or RNA interference against Atg5. (i) Baicalin induced TRAF2 degradation in M2-like macrophage but not in M1 phenotype. (j) Degradation of TRAF2 induced by baicalin may be dependent on autophagy-associated lysosomal pathway. TAM treated with or without baicalin was fixed and stained with TRAF2 antibody. Lysosome was stained with Lysotracker Green for $30 \mathrm{~min}$. It was observed that TRAF2 was translocated to lysosome after treatment of baicalin in TAM (magnification: x60). (k) p62 cargo protein may dominate selective autophagic degradation of TRAF2 in baicalin-treated TAM. TAM was treated with or without baicalin in the presence of lysosome inhibitor leupeptin. Co-immunoprecipitation assay was conducted with TRAF2 antibody and association of p62 with TRAF2 was detected by immunoblotting. Recruitment of p62 to TRAF2 upon baicalin treatment was observed 
macrophage, ${ }^{62}$ which is consistent with our observation that RelB and p52 highly expressed in M1-like but not M2-like macrophage. A positive feedback loop in RelB pathway in baicalin-treated TAM resembles classical activation of macrophages into M1-like phenotype.

In conclusion, our data postulate that the tumour suppressive effect of baicalin was mediated by re-education of TAMs away from M2-like, towards tumour inhibiting M1-like phenotype. This effect was regulated by activation of RelB/p52 pathway via TRAF2 lysosomal degradation-dependent pathway. Baicalin-induced autophagy was responsible for lysosomal degradation of TRAF2 as well as TAM repolarisation. This study proposes baicalin as a potential immune therapeutic candidate for the treatment of hepatocellular carcinoma.

\section{Materials and Methods \\ Chemical, reagents and antibodies. Baicalin, Bafilomycin $\mathrm{A} 1$ and leupeptin were purchased from Sigma-Aldrich (St. Louis, MO, USA). Antibody against MR was obtained from Abcam (Cambridge, UK); antibody against CD11b, Ly6C, CD115, F4/80, CD86 and CD206 were from eBioscience (CA, USA); antibodies against LC3B and iNOS was purchased from Novus Biologicals (Littleton, CO, USA); Antibodies against phosphor-p100, RelA, IKK $\alpha$, RelB, TRAF2, TRAF3 I $\mathrm{K} \alpha_{\alpha}, \mathrm{GADPH}$, were purchased from Cell Signalling Technology (MA, USA); The FITC-conjugated secondary antibody and LysoTracker Green were from Molecular Probe (Eugene, OR, USA), murine recombinant proteins M-CSF, IFNy and IL4 were from Peprotech (NJ, USA).}

Cells, orthotopic HCC implantation animal model and macrophage removal. In vitro and in vivo models of interaction between HCC cells have been established and used in our previous studies. ${ }^{36}$ HCC cell line MHCC97L cells were provided by Professor Man Kwan in Department of Surgery, University of Hong Kong, and were maintained in DMEM supplemented with $10 \%$ fetal bovine serum. Murine orthotopic HCC model was established on BALB/cAnN-nu athymic mice. Removal of macrophage from mice was conducted as indicated in Figure 1a. In brief, 2 days before the implantation of HCC generated by MHCC97L cells, mice in macrophage-removal group ( $\mathrm{M} \Phi-)$ was given clodronate liposome via intraperitoneal injection once $(0.1 \mathrm{ml} / 10 \mathrm{~g}$, ClodronateLiposomes.com, the Netherlands) to deplete macrophage from mice. Macrophage removal was then conducted throughout the experiment by liposomal clodronate treatment every 4 days. Mice in macrophage-presenting groups $(\mathrm{M} \Phi+)$ received equal volume of PBS liposome as control. A small cube $\left(\sim \mathrm{mm}^{3}\right)$ of HCC tumour was implanted into the left lobe of mice liver. Presence of liver tumour was checked 1 week after implantation by laparotomy. Mice in macrophage-presenting groups were then given baicalin ( $50 \mathrm{mg} / \mathrm{kg}$ per 2 days) or equal volume of PBS $(n=4)$. All mice were sacrificed after 5 weeks of treatment. The study protocols were approved by the Committee on the Use of Live Animals in Teaching and Research (CULATR) of The University of Hong Kong, Hong Kong (reference number: 2809-12). Size of the tumour $\left(\mathrm{mm}^{3}\right)$ was measured as (length $\times$ width $)^{2} / 2$. The myeloid cells from bone marrow and circulating system were isolated for further flow cytometry analysis.

Statistical analysis. Studies were performed in triplicate except particular notice in figures. Data were expressed as mean \pm S.D. Statistical significance were analysed using one-way ANOVA followed by Newman-Keuls post hoc test, and $P$-value $<0.05$ considered as significant.

Other Materials and Methods were present in Supplementary Methods.

\section{Conflict of Interest}

The authors declare no conflict of interest.

Acknowledgements. The study was financially supported by grants from the research council of The University of Hong Kong (project codes: 104001764, 104002320, 104002889 and 104003422), the University Grants Committee (UGC) of Hong Kong, HKSAR (project code: 766211), Wong's Donation on Modern Oncology of Chinese Medicine (project code: 200006276), Gala Family Trust (project code: 200007008), Government-Matching Grant Scheme (project code: 207060411) and
Hong Kong Jockey Club Charities Trust (HKJCCT) for funding the project of 'R\&D Laboratory for Testing of Chinese Medicines'. We are grateful to Professor Yung-Chi Cheng for his advice and also would like to thank Mr. Keith Wong, Ms. Cindy Lee, Mr. Alex Shek and Faculty Core Facility for their technical supports.

1. American Cancer Society. Cancer Treatment and Survivorship Facts \& Figures 2014-2015. American Cancer Society: Atlanta, 2014.

2. American Cancer Society. Global Cancer Facts \& Figures, 2nd edn. American Cancer Society: Atlanta, 2011.

3. Yeung OW, Lo C, Ling C, Qi X, Geng W, Li C et al. Alternatively activated (M2) macrophages promote tumour growth and invasiveness in hepatocellular carcinoma. $J$ Hepatol 2014; 62 : 607-616.

4. Sprinzl MF, Puschnik A, Schlitter AM, Schad A, Ackermann K, Esposito I et al. Sorafenib inhibits macrophage-induced growth of hepatoma cells by interference with insulin-like growth factor-1 secretion. J Hepatol 2015; 62: 863-870.

5. Wang $\mathrm{N}$, Liang $\mathrm{H}$, Zen K. Molecular mechanisms that influence the macrophage m1-m2 polarization balance. Front Immunol 2014; 5: 614.

6. Biswas SK, Mantovani A. Macrophage plasticity and interaction with lymphocyte subsets: cancer as a paradigm. Nat Immunol 2010; 11: 889-896.

7. Ruffell B, Affara NI, Coussens LM. Differential macrophage programming in the tumor microenvironment. Trends Immunol 2012; 33: 119-126.

8. Solinas G, Germano G, Mantovani A, Allavena P. Tumor-associated macrophages (TAM) as major players of the cancer-related inflammation. J Leukoc Biol 2009; 86: 1065-1073.

9. Quail DF, Joyce JA. Microenvironmental regulation of tumor progression and metastasis Nat Med 2013; 19: 1423-1437.

10. Tang $\mathrm{X}$, Mo $\mathrm{C}$, Wang $\mathrm{Y}$, Wei $\mathrm{D}$, Xiao $\mathrm{H}$. Anti-tumour strategies aiming to target tumourassociated macrophages. Immunology 2013; 138: 93-104.

11. Capece D, Fischietti M, Verzella D, Gaggiano A, Cicciarelli G, Tessitore A et al. The inflammatory microenvironment in hepatocellular carcinoma: a pivotal role for tumorassociated macrophages. Biomed Res Int 2013; 2013: 187204.

12. Deng $X$, Zhang $P$, Liang T, Deng $S$, Chen $X$, Zhu L. Ovarian cancer stem cells induce the $M 2$ polarization of macrophages through the PPARgamma and NF-kappaB pathways. Int $\mathrm{J} \mathrm{Mol}$ Med 2015; 36: 449-454.

13. Sag D, Cekic C, Wu R, Linden J, Hedrick CC. The cholesterol transporter ABCG1 links cholesterol homeostasis and tumour immunity. Nat Commun 2015; 6: 6354.

14. Devaraj S, Jialal I. C-reactive protein polarizes human macrophages to an $M 1$ phenotype and inhibits transformation to the M2 phenotype. Arterioscler Thromb Vasc Biol 2011; 31: 1397-1402.

15. Peng J, Tsang JY, Li D, Niu N, Ho DH, Lau KF et al. Inhibition of TGF-beta signaling in combination with TLR7 ligation re-programs a tumoricidal phenotype in tumor-associated macrophages. Cancer Lett 2013; 331: 239-249.

16. Guiducci C, Vicari AP, Sangaletti S, Trinchieri G, Colombo MP. Redirecting in vivo elicited tumor infiltrating macrophages and dendritic cells towards tumor rejection. Cancer Res 2005; 65: 3437-3446.

17. Vrancic M, Banjanac M, Nujic K, Bosnar M, Murati T, Munic V et al. Azithromycin distinctively modulates classical activation of human monocytes in vitro. Br J Pharmacol 2012; 165: 1348-1360.

18. Coscia M, Quaglino E, lezzi M, Curcio C, Pantaleoni F, Riganti C et al. Zoledronic acid repolarizes tumour-associated macrophages and inhibits mammary carcinogenesis by targeting the mevalonate pathway. J Cell Mol Med 2010; 14: 2803-2815.

19. Jang JY, Lee JK, Jeon YK, Kim CW. Exosome derived from epigallocatechin gallate treated breast cancer cells suppresses tumor growth by inhibiting tumor-associated macrophage infiltration and M2 polarization. BMC cancer 2013; 13: 421.

20. Kono Y, Kawakami S, Higuchi Y, Yamashita F, Hashida M. In vitro evaluation of inhibitory effect of nuclear factor-kappaB activity by small interfering RNA on pro-tumor characteristics of M2-like macrophages. Biol Pharm Bull 2014; 37: 137-144.

21. Saccani A, Schioppa T, Porta C, Biswas SK, Nebuloni M, Vago L et al. p50 nuclear factorkappaB overexpression in tumor-associated macrophages inhibits $M 1$ inflammatory responses and antitumor resistance. Cancer Res 2006; 66: 11432-11440.

22. Biswas SK, Gangi L, Paul S, Schioppa T, Saccani A, Sironi M et al. A distinct and unique transcriptional program expressed by tumor-associated macrophages (defective NF-kappaB and enhanced IRF-3/STAT1 activation). Blood 2006; 107: 2112-2122.

23. Mancino A, Lawrence T. Nuclear factor-kappaB and tumor-associated macrophages. Clin Cancer Res 2010; 16: 784-789.

24. Hagemann T, Lawrence T, McNeish I, Charles KA, Kulbe H, Thompson RG et al. "Re-educating" tumor-associated macrophages by targeting NF-kappaB. J Exp Med 2008; 205: 1261-1268.

25. Chen PC, Cheng HC, Wang J, Wang SW, Tai HC, Lin CW et al. Prostate cancer-derived CCN3 induces M2 macrophage infiltration and contributes to angiogenesis in prostate cancer microenvironment. Oncotarget 2014; 5: 1595-1608.

26. Kono Y, Kawakami S, Higuchi Y, Maruyama K, Yamashita F, Hashida M. Tumour-associated macrophages targeted transfection with NF-kappaB decoy/mannose-modified bubble lipoplexes inhibits tumour growth in tumour-bearing mice. J Drug Target 2014; 22: 439-449. 
27. Chan G, Bivins-Smith ER, Smith MS, Yurochko AD. NF-kappaB and phosphatidylinosito 3-kinase activity mediates the HCMV-induced atypical M1/M2 polarization of monocytes. Virus Res 2009; 144: 329-333.

28. Chiu YW, Lin TH, Huang WS, Teng CY, Liou YS, Kuo WH et al. Baicalein inhibits the migration and invasive properties of human hepatoma cells. Toxicol Appl Pharmacol 2011 255: 316-326.

29. Motoo Y, Sawabu N. Antitumor effects of saikosaponins, baicalin and baicalein on human hepatoma cell lines. Cancer Lett 1994; 86: 91-95.

30. Yang J, Yang X, Chu Y, Li M. Identification of Baicalin as an immunoregulatory compound by controlling $\mathrm{T}(\mathrm{H}) 17$ cell differentiation. PLoS One 2011; 6: e17164

31. Kim ME, Kim HK, Park HY, Kim DH, Chung HY, Lee JS. Baicalin from Scutellaria baicalensis impairs Th1 polarization through inhibition of dendritic cell maturation. J Pharmacol Sci2013 121: $148-156$.

32. Gong SQ, Sun W, Wang M, Fu YY. Role of TLR4 and TCR or BCR against baicalin-induced responses in T and B cells. Int Immunopharmacol 2011; 11: 2176-2180.

33. Wang N, Feng Y, Tan HY, Cheung F, Hong M, Lao L et al. Inhibition of eukaryotic elongation factor-2 confers to tumor suppression by a herbal formulation Huanglian-Jiedu decoction in human hepatocellular carcinoma. J Ethnopharmacol 2015; 164: 309-318.

34. Zhang X, Tang X, Liu H, Li L, Hou Q, Gao J. Autophagy induced by baicalin involves downregulation of CD147 in SMMC-7721 cells in vitro. Oncol Rep 2012; 27: 1128-1134.

35. Tsang CM, Cheung KC, Cheung YC, Man K, Lui VW, Tsao SW et al. Berberine suppresses Id-1 expression and inhibits the growth and development of lung metastases in hepatocellular carcinoma. Biochim Biophys Acta 2015; 1852: 541-551.

36. Yeung OW, Lo CM, Ling CC, Qi X, Geng W, Li CX et al. Alternatively activated (M2) macrophages promote tumour growth and invasiveness in hepatocellular carcinoma. $J$ Hepatol 2015; 62: 607-616.

37. Obeid E, Nanda R, Fu YX, Olopade OI. The role of tumor-associated macrophages in breast cancer progression (review). Int J Oncol 2013; 43: 5-12.

38. Murray PJ, Wynn TA. Protective and pathogenic functions of macrophage subsets. Nat Rev Immunol 2011; 11: 723-737.

39. Rolny C, Mazzone M, Tugues S, Laoui D, Johansson I, Coulon C et al. HRG inhibits tumo growth and metastasis by inducing macrophage polarization and vessel normalization through downregulation of PIGF. Cancer Cell 2011; 19: 31-44.

40. Yin Y, Huang X, Lynn KD, Thorpe PE. Phosphatidylserine-targeting antibody induces $M 1$ macrophage polarization and promotes myeloid-derived suppressor cell differentiation. Cancer Immunol Res 2013; 1: 256-268.

41. Lin C, Tsai SC, Tseng MT, Peng SF, Kuo SC, Lin MW et al. AKT serine/threonine protein kinase modulates baicalin-triggered autophagy in human bladder cancer T24 cells. Int J Oncol 2013; 42: 993-1000.

42. Gaetano C, Massimo L, Alberto M. Control of iron homeostasis as a key component of macrophage polarization. Haematologica 2010; 95: 1801-1803.

43. Yang H, Zhang Y, Wu M, Li J, Zhou W, Li G et al. Suppression of ongoing experimental autoimmune myasthenia gravis by transfer of RelB-silenced bone marrow dentritic cells is associated with a change from a Thelper Th17/Th1 to a Th2 and FoxP3+ regulatory T-cell profile. Inflamm Res 2010; 59: 197-205.

44. O'Sullivan BJ, MacDonald KP, Pettit AR, Thomas R. RelB nuclear translocation regulates B cell MHC molecule, CD40 expression, and antigen-presenting cell function. Proc Natl Acad Sci USA 2000; 97: 11421-11426.

45. Oeckinghaus A, Ghosh S. The NF-kappaB family of transcription factors and its regulation Cold Spring Harb Perspect Biol 2009; 1: a000034

46. Bijli KM, Fazal F, Rahman A. Regulation of Rela/p65 and endothelial cell inflammation by proline-rich tyrosine kinase 2. Am J Respir Cell Mol Biol 2012; 47: 660-668.

47. Chang $\mathrm{CP}, \mathrm{Su}$ YC, Hu CW, Lei HY. TLR2-dependent selective autophagy regulates NF-kappaB lysosomal degradation in hepatoma-derived M2 macrophage differentiation. Cell Death Differ 2013; 20: 515-523.

48. De Palma M, Lewis CE. Macrophage regulation of tumor responses to anticancer therapies Cancer Cell 2013; 23: 277-286.
49. Zhu XD, Zhang JB, Zhuang PY, Zhu HG, Zhang W, Xiong YQ et al. High expression of macrophage colony-stimulating factor in peritumoral liver tissue is associated with poor survival after curative resection of hepatocellular carcinoma. J Clin Oncol 2008; 26 : 2707-2716.

50. Zhang W, Zhu XD, Sun HC, Xiong YQ, Zhuang PY, Xu HX et al. Depletion of tumor-associated macrophages enhances the effect of sorafenib in metastatic liver cancer models by antimetastatic and antiangiogenic effects. Clin Cancer Res 2010; 16: $3420-3430$.

51. Wan S, Zhao E, Kryczek I, Vatan L, Sadovskaya A, Ludema G et al. Tumor-associated macrophages produce interleukin 6 and signal via STAT3 to promote expansion of human hepatocellular carcinoma stem cells. Gastroenterology 2014; 147: 1393-1404.

52. Martinez FO, Gordon S. The M1 and M2 paradigm of macrophage activation: time for reassessment. F1000Prime Rep 2014; 6: 13

53. Chen N, Nishio N, Ito S, Tanaka Y, Sun Y, Isobe KI. Growth arrest and DNA damageinducible protein (GADD34) enhanced liver inflammation and tumorigenesis in a diethylnitrosamine (DEN)-treated murine model. Cancer Immunol Immunother 2015; 64: 777-789.

54. Li X, Yang Y, Ashwell JD. TNF-RII and C-IAP1 mediate ubiquitination and degradation of TRAF2. Nature 2002; 416: 345-347.

55. Dupoux A, Cartier J, Cathelin S, Filomenko R, Solary E, Dubrez-Daloz L. cIAP1-dependent TRAF2 degradation regulates the differentiation of monocytes into macrophages and their response to CD40 ligand. Blood 2009; 113: 175-185.

56. Li L, Soetandyo N, Wang Q, Ye Y. The zinc finger protein A20 targets TRAF2 to the lysosomes for degradation. Biochim Biophys Acta 2009; 1793: 346-353.

57. Johansen T, Lamark T. Selective autophagy mediated by autophagic adapter proteins. Autophagy 2011; 7: 279-296.

58. Goktuna SI, Canli O, Bollrath J, Fingerle AA, Horst D, Diamanti MA et al. Greten, IKKalpha promotes intestinal tumorigenesis by limiting recruitment of M1-like polarized myeloid cells. Cell Rep 2014; 7: 1914-1925.

59. Sun SC. Non-canonical NF-kappaB signaling pathway. Cell Res 2011; 21: 71-85.

60. Neumann M, Wohlleben G, Chuvpilo S, Kistler B, Wirth T, Serfling E et al. CD40, but not lipopolysaccharide and anti-IgM stimulation of primary $B$ lymphocytes, leads to a persistent nuclear accumulation of RelB. J Immunol 1996; 157: 4862-4869.

61. Guan H, Hou S, Ricciardi RP. DNA binding of repressor nuclear factor-kappaB p50/p50 depends on phosphorylation of Ser337 by the protein kinase A catalytic subunit. J Biol Chem 2005; 280: 9957-9962.

62. Li T, Morgan MJ, Choksi S, Zhang Y, Kim YS, Liu ZG. MicroRNAs modulate the noncanonical transcription factor NF-kappaB pathway by regulating expression of the kinase IKKalpha during macrophage differentiation. Nat Immunol 2010; 11: 799-805.

63. Bosisio D, Marazzi I, Agresti A, Shimizu N, Bianchi ME, Natoli G. A hyper-dynamic equilibrium between promoter-bound and nucleoplasmic dimers controls NF-kappaBdependent gene activity. EMBO J 2006; 25: 798-810.

64. Basak S, Shih VF, Hoffmann A. Generation and activation of multiple dimeric transcription factors within the NF-kappaB signaling system. Mol Cell Biol 2008; 28: 3139-3150.

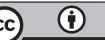

Cell Death and Disease is an open-access journal published by Nature Publishing Group. This work is licensed under a Creative Commons Attribution 4.0 International License. The images or other third party material in this article are included in the article's Creative Commons license, unless indicated otherwise in the credit line; if the material is not included under the Creative Commons license, users will need to obtain permission from the license holder to reproduce the material. To view a copy of this license, visit http://creativecommons.org/licenses/by/4.0/ 